\title{
Split clique graph complexity
}

\author{
Liliana Alcón ${ }^{\mathrm{a}}$, Luerbio Faria ${ }^{\mathrm{c}}$, Celina M.H. de Figueiredo ${ }^{\mathrm{d}, *}$, Marisa Gutierrez ${ }^{\mathrm{b}}$ \\ a Departamento de Matemática, UNLP, Argentina \\ b CONICET, Departamento de Matemática, UNLP, Argentina \\ c Departamento de Matemática, FFP, UERJ, Brazil \\ d Programa de Engenharia de Sistemas e Computação, COPPE, UFRJ, Brazil
}

\section{A R T I C L E I N F O}

\section{Article history:}

Received 8 December 2011

Received in revised form 13 May 2013

Accepted 18 July 2013

Communicated by J. Kratochvil

\section{Keywords:}

Clique graphs

Helly property

NP-complete

Split graphs

\begin{abstract}
A B S T R A C T
A complete set of a graph $G$ is a subset of vertices inducing a complete subgraph. A clique is a maximal complete set. Denote by $\mathcal{C}(G)$ the clique family of $G$. The clique graph of $G$, denoted by $K(G)$, is the intersection graph of $\mathcal{C}(G)$. Say that $G$ is a clique graph if there exists a graph $H$ such that $G=K(H)$. The clique graph recognition problem, a longstanding open question posed in 1971, asks whether a given graph is a clique graph and it was recently proved to be NP-complete even for a graph $G$ with maximum degree 14 and maximum clique size 12 . Hence, if $\mathrm{P} \neq \mathrm{NP}$, the study of graph classes where the problem can be proved to be polynomial, or of more restricted graph classes where the problem remains NP-complete is justified. We present a proof that given a split graph $G=(V, E)$ with partition $(K, S)$ for $V$, where $K$ is a complete set and $S$ is a stable set, deciding whether there is a graph $H$ such that $G$ is the clique graph of $H$ is NP-complete. As a byproduct, we prove that determining whether a given set family admits a spanning family satisfying the Helly property is NP-complete. Our result is optimum in the sense that each vertex of the independent set of our split instance has degree at most 3 , whereas when each vertex of the independent set has degree at most 2 the problem is polynomial, since it is reduced to the problem of checking whether the clique family of the graph satisfies the Helly property. Additionally, we show three split graph subclasses for which the problem is polynomially solvable: the subclass where each vertex of $S$ has a private neighbor, the subclass where $|S| \leqslant 3$, and the subclass where $|K| \leqslant 4$.
\end{abstract}

(C) 2013 Elsevier B.V. All rights reserved.

\section{Introduction}

Consider finite, simple and undirected graphs. $V$ and $E$ denote the vertex set and the edge set of the graph $G$, respectively. A complete set of $G$ is a subset of $V$ inducing a complete subgraph. A clique is a maximal complete set. The clique family of $G$ is denoted by $\mathcal{C}(G)$. The clique graph of $G$ is the intersection graph of $\mathcal{C}(G)$.

The clique operator $K$ assigns to each graph $G$ its clique graph which is denoted by $K(G)$. On the other hand, say that $G$ is a clique graph if $G$ belongs to the image of the clique operator, i.e. if there exists a graph $H$ such that $G=K(H)$.

\footnotetext{
th An extended abstract published in: Proceedings of Graph-Theoretic Concepts in Computer Science, WG 2011, Lecture Notes in Comput. Sci., Springer. This research was partially supported by FAPERJ-Cientistas do Nosso Estado Project, by PROCIENCIA Project, and by CNPq Project PROSUL - Proc. No. 490333/04-4.

* Corresponding author.

E-mail addresses: liliana@mate.unlp.edu.ar (L. Alcón), luerbio@cos.ufrj.br (L. Faria), celina@cos.ufrj.br (C.M.H. de Figueiredo), marisa@mate.unlp.edu.ar (M. Gutierrez).
} 
Clique operator and its image were widely studied. First articles focused on recognizing clique graphs [9,20]. Graphs fixed under the operator $K$ or fixed under the iterated clique operator, $K^{n}$, for some positive integer $n$; and the behavior under these operators of parameters such as number of vertices or diameter were studied in [4,10,11]. For several classes of graphs, the image of the class under the clique operator was characterized [5,12,18,21]; and, in some cases, also the inverse image of the class $[15,19]$. Results of the previous bibliography can be found in the survey [23]. Clique graphs have been much studied as intersection graphs and are included in several books [6,13,17].

The characterization of clique graphs given in [20] proposed the computational complexity of the recognition of clique graphs, a long-standing open question $[6,17,20,23]$ just recently settled as NP-complete $[1,2]$.

A graph is split if its vertex set can be partitioned into a complete set and a stable set. In this paper, we are concerned with the time complexity of the problem of recognizing split clique graphs, for which we establish NP-complete and polynomial results.

\section{SPLIT CLIQUE GRAPH}

INSTANCE: A split graph $G=(V, E)$.

QUESTION: Is there a graph $H$ such that $G=K(H)$ ?

We prove that SPLIT CLIQUE GRAPH is NP-complete. As a byproduct, we prove that a problem about the Helly property is NP-complete. Given a set family $\mathcal{F}=\left(F_{i}\right)_{i \in I}$, say that the sets $F_{i}$ are members of the family, and $F \in \mathcal{F}$ means that $F$ is a member of $\mathcal{F}$. The family is pairwise intersecting if the intersection of any two members is not the empty set. The intersection or total intersection of $\mathcal{F}$ is the set $\bigcap \mathcal{F}=\bigcap_{i \in I} F_{i}$. The family $\mathcal{F}$ has the Helly property, if any pairwise intersecting subfamily has nonempty total intersection. Besides the theoretical interest, the Helly property has applications in many different areas such as optimization and location problems, semantics, coding, computational biology, data bases, image processing and, in special, graph theory where it has been a useful and a natural tool. We refer to [7] for a survey on the Helly property and its complexity aspects.

Given a family of sets $\mathcal{F}$, say that a family $\mathcal{F}^{\prime}$ is a spanning family for $\mathcal{F}$ if: $\bigcup_{F^{\prime} \in \mathcal{F}^{\prime}} F^{\prime}=\bigcup_{F \in \mathcal{F}} F$; for each $F^{\prime} \in \mathcal{F}^{\prime}$, $\left|F^{\prime}\right|>1$; for each $F^{\prime} \in \mathcal{F}^{\prime}$, there exists $F \in \mathcal{F}$ such that $F^{\prime} \subseteq F$; and for each $F \in \mathcal{F}, \bigcup_{F^{\prime} \subseteq F, F^{\prime} \in \mathcal{F}^{\prime}} F^{\prime}=F$.

\section{SPANNING HELLY FAMILY}

INSTANCE: A family of sets $\mathcal{F}$.

QUESTION: Does $\mathcal{F}$ admit a spanning family $\mathcal{F}^{\prime}$ that satisfies the Helly property?

We prove as a corollary that our NP-completeness result yields that sPANNING HeLLY FAMILY is NP-complete even when restricted to the members of the input family $\mathcal{F}$ having cardinality 2 or 3 . Note that the problem is polynomial when all members of $\mathcal{F}$ have cardinality 2. We prove an additional NP-completeness result that implies that SPANNING HELLY FAMILY is NP-complete even when restricted to all members of the input family $\mathcal{F}$ having cardinality 3.

Section 2 contains the NP-completeness results of the problem of recognizing clique graphs when restricted to split graphs: first when restricted to 3-split inputs that admit a split partition where each vertex of the stable set $S$ has degree 2 or 3 ; second further restricted to 3 -split $_{3}$ inputs that admit a split partition where each vertex of the stable set $S$ has degree exactly 3 . Section 3 contains the polynomial results obtained by restricting the size of the complete set $K$ or of the stable set $S$. Section 4 concludes the paper and proposes related open problems.

\section{NP-complete split clique graph classes}

Theorem 1 is a well known characterization of Clique Graphs. The edge with end vertices $u$ and $v$ is represented by $u v$. We say that the complete set $C$ covers the edge $u v$ when $u$ and $v$ belong to $C$. A complete set edge cover of a graph $G$ is a family of complete sets of $G$ covering all edges of $G$.

Theorem 1. (See Roberts and Spencer [20].) G is a clique graph if and only if there exists a complete set edge cover of $G$ satisfying the Helly property.

Notice that for any graph $G$ the clique family $\mathcal{C}(G)$ is a complete set edge cover of $G$, but, in general, this family does not satisfy the Helly property. Graphs such that $\mathcal{C}(G)$ satisfies the Helly property are called clique-Helly graphs. It follows from Theorem 1 that every clique-Helly graph is a clique graph. In [22], clique-Helly graphs are characterized and a polynomial-time algorithm for their recognition is presented. Lemma 2 extends that result and leads to a polynomial-time algorithm to check if a given complete set edge cover of a graph satisfies the Helly property which in turn yields that CLIQUE GRAPH is in NP [1,2].

A triangle is a complete set with exactly 3 vertices. The set of triangles of $G$ is denoted $T(G)$. Let $\mathcal{F}$ be a complete set edge cover of $G$ and $T$ a triangle, and denote by $\mathcal{F}_{T}$ the subfamily of $\mathcal{F}$ formed by all the members containing at least two vertices of $T$. 
Lemma 2. (See Alcón and Gutierrez [3].) Let $\mathcal{F}$ be a complete set edge cover of $G$. The following conditions are equivalent:

(i) $\mathcal{F}$ has the Helly property.

(ii) For every $T \in T(G)$, the subfamily $\mathcal{F}_{T}$ has the Helly property.

(iii) For every $T \in T(G)$, the subfamily $\mathcal{F}_{T}$ has nonempty intersection, this means $\bigcap \mathcal{F}_{T} \neq \emptyset$.

A graph admits a complete set edge cover with the Helly property if and only if the graph admits a complete set edge cover with the Helly property such that no member is contained in another; such cover is called an RS-family of the graph. Thus Theorem 1 is equivalent to the following simpler statement: $G$ is a clique graph if and only if $G$ admits an RS-family. The following properties are stated and proved by Roberts and Spencer [20].

Lemma 3. (See Lemma 1 and Theorem 3 of [20].) Let $\mathcal{F}$ be an RS-family of a graph $G$. Then $\mathcal{F}$ contains a complete set of size 2 if and only if this complete set is a clique of $G$. If a triangle $T$ is a clique of $G$, then $T$ is a member of $\mathcal{F}$.

We show that SPLIT CLIQUE GRAPH is NP-complete by a reduction from the following version of the 3-satisfiability problem with at most 3 occurrences per variable [14]. Let $U=\left\{u_{i}, 1 \leqslant i \leqslant n\right\}$ be a set of boolean variables. A literal is either a variable $u_{i}$ or its complement $\overline{u_{i}}$. A clause over $U$ is a set of literals. Let $C=\left\{c_{j}, 1 \leqslant j \leqslant m\right\}$ be a collection of clauses over $U$. We say that variable $u_{i}$ occurs in clause $c_{j}$ (and then in $C$ ) if $u_{i}$ or $\overline{u_{i}} \in c_{j}$. We say that variable $u_{i}$ occurs in clause $c_{j}$ as literal $u_{i}$ (or that literal $u_{i}$ occurs in $c_{j}$ ) if $u_{i} \in c_{j}$, and as literal $\overline{u_{i}}$ (or that literal $\overline{u_{i}}$ occurs in $c_{j}$ ) if $\overline{u_{i}} \in c_{j}$.

$3 \mathrm{SAT}_{\overline{3}}$

INSTANCE: $I=(U, C)$, where $U=\left\{u_{i}, 1 \leqslant i \leqslant n\right\}$ is a set of boolean variables, and $C=\left\{c_{j}, 1 \leqslant j \leqslant m\right\}$ a set of clauses over $U$

such that each clause has two or three variables, each variable occurs at most three times in $C$.

QUESTION: Is there a truth assignment for $U$ such that each clause in $C$ has at least one true literal?

In order to reduce $3 \mathrm{SAT}_{\overline{3}}$ to SPLIT CLIQUE GRAPH, we need to construct in polynomial time a particular instance $G_{I}$ of SPLIT CLIQUE GRAPH from a generic instance $I=(U, C)$ of $3 \mathrm{SAT}_{\overline{3}}$, in such a way that the constructed graph $G_{I}$ is a clique graph if and only if $C$ is satisfiable. The particular instance $G_{I}$ is a 3-split graph and we first characterize 3-split clique graphs.

\subsection{3-Split graphs}

A split graph admits a split partition of its vertex set into a complete set $K$ and a stable set $S$. The family of cliques of a split graph with split partition $(K, S)$ is composed by the closed neighborhood $N[s]$, for each $s \in S$, and the complete set $K$ if it is not contained in $N[s]$, for $s \in S$. An $\ell$-cone is an $(\ell+1)$-clique containing a vertex of $S$ that is called its extreme vertex and the remaining $\ell$ vertices are in $K$ composing the basis of the cone. Note that any 2 -cone is a clique and so by Lemma 3 forced to belong to any RS-family of a split clique graph.

Lemma 4. Let $G$ be a split graph with split partition $(K, S)$.

(i) If there exist three 2-cones whose bases form a triangle then $G$ is not a clique graph.

(ii) If there exist one 3 -cone and three 2 -cones whose bases are respectively $\{x, y, w\},\{x, z\},\{y, z\}$ and $\{w, z\}$, being $x, y, z, w$ vertices of $K$, then $G$ is not a clique graph.

Proof. Assume there exists an RS-family $\mathcal{F}$ of $G$. Since the three 2-cones are cliques of $G$, by Lemma 3, they are members of $\mathcal{F}$. Since their bases form a triangle, the three 2-cones do not satisfy the Helly property, a contradiction.

To prove the second statement, let $N\left[s_{1}\right]=\left\{s_{1}, x, y, w\right\}, N\left[s_{2}\right]=\left\{s_{2}, x, z\right\}, N\left[s_{3}\right]=\left\{s_{3}, y, z\right\}$ and $N\left[s_{4}\right]=\left\{s_{4}, z, w\right\}$ be cones of $G$ and suppose for a contradiction that $G$ is a clique graph. Thus there exists an RS-family $\mathcal{F}$ of $G$. Since $N\left[s_{2}\right]$, $N\left[s_{3}\right]$ and $N\left[s_{4}\right]$ must be members of $\mathcal{F}$, any other member $F$ covering the edge $x y$ or the edge $y w$ must contain $z$ in order to satisfy the Helly property. But, since $N\left[s_{1}\right]$ is a complete set of 4 vertices, if $F^{\prime}$ is a member of $\mathcal{F}$ covering $s_{1} y$ then $x \in F^{\prime}$ or $w \in F^{\prime}$. In any case $z \in F^{\prime}$, which is a contradiction because $s_{1}$ is not adjacent to $z$.

A 3-split graph admits a split partition where each vertex of the stable set $S$ has degree 2 or 3 , in this case $(K, S)$ is called a 3-split partition. A triangle of a 3-cone is any triangle that contains the extreme vertex of the cone. The two vertices of the triangle belonging to the basis of the 3-cone are the basis of the triangle. A 3-cone has exactly three triangles. The edge induced by the basis of a 2-cone or by the basis of a triangle of a 3-cone is also called basis.

Theorem 5. Let $G$ be a 3-split graph with 3-split partition $(K, S)$. The following are equivalent:

(1) $G$ is a clique graph;

(2) There is a family composed by K, each 2-cone and exactly two triangles of each 3-cone, that is an RS-family of G; 
(3) There is a family composed by the basis of each 2-cone and the bases of exactly two triangles of each 3-cone, that satisfies the Helly property;

(4) There is a set of edges of $K$ composed by the basis of each 2-cone and the bases of exactly two triangles of each 3-cone, that induces a triangle-free subgraph of $G[K]$.

Proof. 1 implies 2: Let $\mathcal{F}$ be an RS-family of $G$. Assume $K$ is not a member of $\mathcal{F}$. Consider $\mathcal{F}^{\prime}$ the family obtained from $\mathcal{F}$ by the addition of member $K$ and by the removal of complete sets $K^{\prime}$ that satisfy $K^{\prime}$ is a member of $\mathcal{F}$ and $K^{\prime} \subset K$. Suppose there exists a pairwise intersecting subfamily of $\mathcal{F}^{\prime}$ without a common vertex. It is clear this subfamily must contain $K$, since the original RS-family $\mathcal{F}$ has the Helly property. Let $F_{1}, F_{2}, \ldots, F_{\ell}, K$ be the pairwise intersecting subfamily without a common vertex. Observe that $\ell \geqslant 2$. Since $F_{1}, F_{2}, \ldots, F_{\ell}$ are members of $\mathcal{F}$, they have a common vertex $s$. It is clear $s$ is not in $K$, and so $s \in S$. In case $s$ has degree 2 , say $N(s)=\{x, y\}$, then $F_{1}=\{s, x\}$ and $F_{2}=\{s, y\}$ but this contradicts Lemma 3 since $F_{1}$ and $F_{2}$ are not cliques of $G$. Hence, $N(s)=\{x, y, z\}$ and the assumption that $F_{1}, F_{2}, \ldots, F_{\ell}$ have no common vertex in $K$ forces $\ell=3, F_{1}=\{s, y, z\}, F_{2}=\{x, s, z\}$ and $F_{3}=\{x, y, s\}$. Note that $F_{1}, F_{2}$ and $F_{3}$ are the three triangles containing vertex $s$. Now we can eliminate one of these three triangles from $\mathcal{F}^{\prime}$, the remaining two triangles have a common vertex in $K$ and cover the same set of edges as $\mathcal{F}^{\prime}$. Observe that in case we have another intersecting subfamily in $\mathcal{F}^{\prime}$ without a common vertex, it must be the three triangles of another 3-cone. We repeat the same reasoning for each such pairwise intersecting subfamily to obtain an RS-family containing $K$.

So we may assume that $K$ is a member of the RS-family $\mathcal{F}$. Recall that each 2-cone is a clique and must be a member of $\mathcal{F}$. Let $C_{s}=\{s, x, y, z\}$ be a 3-cone with extreme $s$ and basis $T=\{x, y, z\}$. In order to cover the edges incident to $s, \mathcal{F}$ must contain exactly two triangles of $C_{s}$ or must contain the 3-cone $C_{s}$ itself. Suppose $C_{s} \in \mathcal{F}$. Note that no other member of $\mathcal{F}$ contains $s$. By Lemma 2, let $u_{T} \in \bigcap \mathcal{F}_{T}$. Since $u_{T} \in K \cap C_{s}=T$, we may assume $u_{T}=y$. Consider $\mathcal{F}^{\prime}$ obtained from $\mathcal{F}$ by the removal of cone $C_{s}$ and the addition of triangles $\{y, x, s\}$ and $\{y, z, s\}$. We claim $\mathcal{F}^{\prime}$ has the Helly property. Indeed, first suppose $F_{1}, F_{2}, \ldots, F_{\ell},\{y, x, s\}$ is a pairwise intersecting subfamily of $\mathcal{F}^{\prime}$ without a common vertex and that $\{y, z, s\}$ is not a member of the subfamily. Since $F_{i} \cap\{y, x, s\} \neq \emptyset$ and $s \notin F_{i}$, we may assume $x \in F_{1}$ and $y \notin F_{1}, x \notin F_{2}$ and $y \in F_{2}$. Since $F_{1}, F_{2}, C_{s}$ are pairwise intersecting members of $\mathcal{F}$, we must have $z=F_{1} \cap F_{2} \cap C_{s}$. Now $z, x \in F_{1}$ implies $F_{1} \in \mathcal{F}_{T}$, so $y \in F_{1}$, a contradiction. Now suppose $F_{1}, F_{2}, \ldots, F_{\ell},\{y, x, s\},\{y, z, s\}$ is a pairwise intersecting subfamily of $\mathcal{F}^{\prime}$ without a common vertex. We may assume $y \notin F_{1}$, but $F_{1} \cap\{y, x, s\} \neq \emptyset, F_{1} \cap\{y, z, s\} \neq \emptyset$ and $s \notin F_{1}$, thus $F_{1} \in \mathcal{F}_{T}$, again leading to a contradiction.

2 implies 3: Assume $G$ admits an RS-family $\mathcal{F}$ composed by $K$, each 2-cone and exactly two triangles of each 3-cone. Let $\mathcal{B}$ be the family whose members are the bases of the cones and of the triangle members of $\mathcal{F}$. Note that each pairwise intersecting subfamily $\mathcal{B}^{\prime}$ of $\mathcal{B}$ corresponds to a pairwise intersecting subfamily $\mathcal{F}^{\prime}$ of $\mathcal{F}$. Since $\mathcal{F}^{\prime}$ plus the member $K$ is also pairwise intersecting, $\mathcal{F}^{\prime}$ has a common vertex that belongs to $K$. Clearly this is also a common vertex for the subfamily $\mathcal{B}^{\prime}$ of $\mathcal{B}$

3 implies 4: Assume $G$ admits a family $\mathcal{B}$ composed by the basis of each 2-cone and the bases of exactly two triangles of each 3-cone that satisfies the Helly property. Let $\mathcal{E}$ be the corresponding set of edges which are bases of the 2-cones and bases of exactly two triangles of each 3-cone. The Helly property satisfied by $\mathcal{B}$ implies that $\mathcal{E}$ induces a triangle-free subgraph of $G[K]$.

4 implies 1: Let $\mathcal{E}$ be a set of edges composed by the bases of the 2-cones and the bases of exactly two triangles of each 3-cone that induces a triangle-free subgraph of $G[K]$. Let $e=x y$ be an edge of the family $\mathcal{E}$. Call $S_{e}=\{s \in S \mid\{x, y\} \subseteq N(s)\}$. Observe that: (a) if $s$ is the extreme vertex of a 2-cone then $s$ belongs to exactly one set $S_{e}$; (b) if $s$ is the extreme vertex of a 3-cone then $s$ belongs to exactly two sets $S_{e}$. Consider the complete set family $\mathcal{F}$ whose members are $K$ and the triangles $T_{e, s}$, where $e \in \mathcal{E}$ and $s \in S_{e}$. By (a) and (b), if a subfamily of triangles $T_{e, s}$ is pairwise intersecting then the corresponding family of edges $e$ is pairwise intersecting. Since, by hypothesis, the family of edges does not induce a triangle, then they have a common vertex in $K$, which implies $\mathcal{F}$ is an RS-family.

The set of edges defined in statement 4 of Theorem 5 is called an RS-basis of a 3-split clique graph.

\subsection{Construction of $G_{I}$ from $I=(U, C)$}

Let $I=(U, C)$ be any instance of $3 \mathrm{SAT}_{\overline{3}}$. We assume with no loss of generality that each variable occurs two or three times in $C$, and no variable occurs twice in the same clause. In addition, if variable $u_{i}$ occurs twice in $C$, then we assume it is once as literal $u_{i}$ and once as literal $\overline{u_{i}}$; and if variable $u_{i}$ occurs three times in $C$, then we assume it is once as literal $u_{i}$ and twice as literal $\overline{u_{i}}$.

For each variable $u_{i}$, let $j_{i}$ be the subindex of the unique clause where variable $u_{i}$ occurs as literal $u_{i}$; and $\bar{J}_{i}=\{j \mid$ literal $\overline{u_{i}}$ occurs in $c_{j}$ \}.

For each clause $c_{j}$ with $\left|c_{j}\right|=3$, let $I_{j}=\left\{i \mid\right.$ variable $u_{i}$ occurs in $\left.c_{j}\right\}$; and for each clause $c_{j}$ with $\left|c_{j}\right|=2$, let $I_{j}=\{i \mid$ variable $u_{i}$ occurs in $\left.c_{j}\right\} \cup\{n+1\}$. Notice that in any case $\left|I_{j}\right|=3$.

We construct from instance $I=(U, C)$ a graph $G_{I}=(V, E)$ as follows. 


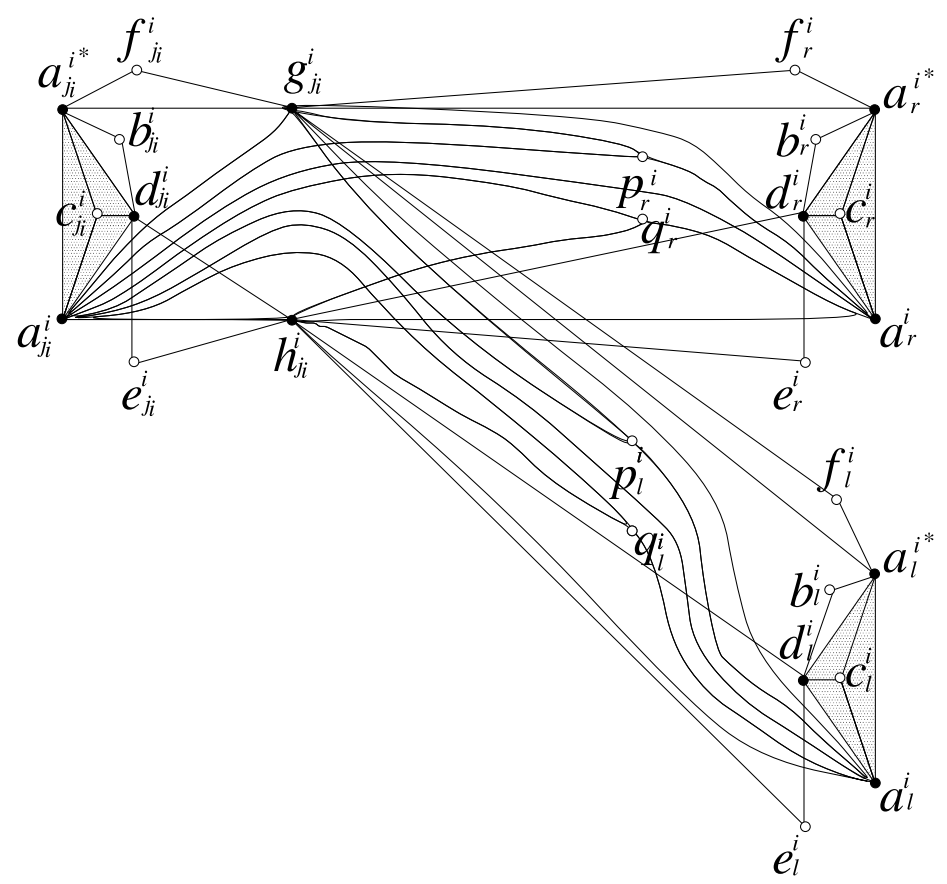

Fig. 1. Graph $T_{i}$ corresponding to a variable $u_{i}$, with $\bar{J}_{i}=\{r, l\}$.

The vertex set $V$ is the union:

$$
\begin{aligned}
V= & \bigcup_{1 \leqslant i \leqslant n}\left\{a_{j_{i}}^{i}, b_{j_{i}}^{i}, c_{j_{i}}^{i}, d_{j_{i}}^{i}, e_{j_{i}}^{i}, f_{j_{i}}^{i}, g_{j_{i}}^{i}, h_{j_{i}}^{i}\right\} \\
& \cup \bigcup_{1 \leqslant i \leqslant n} \bigcup_{j \in \bar{J}_{i}}\left\{a_{j}^{i}, b_{j}^{i}, c_{j}^{i}, d_{j}^{i}, e_{j}^{i}, f_{j}^{i}, p_{j}^{i}, q_{j}^{i}\right\} \\
& \cup \underset{1 \leqslant j \leqslant m,\left|c_{j}\right|=2}{\bigcup}\left\{a_{j}^{n+1}, c_{j}^{n+1}, d_{j}^{n+1}\right\} .
\end{aligned}
$$

The complete set is:

$$
K=\bigcup_{1 \leqslant i \leqslant n}\left\{a_{j_{i}}^{i}, d_{j_{i}}^{i}, g_{j_{i}}^{i}, h_{j_{i}}^{i}\right\} \cup \bigcup_{1 \leqslant i \leqslant n} \bigcup_{j \in \bar{J}_{i}}\left\{a_{j}^{i}, d_{j}^{i}\right\} \cup \underset{1 \leqslant j \leqslant m,\left|c_{j}\right|=2}{\bigcup}\left\{a_{j}^{n+1}, d_{j}^{n+1}\right\}
$$

and the stable set is composed by the remaining vertices $S=V \backslash K$ :

$$
S=\bigcup_{1 \leqslant i \leqslant n}\left\{b_{j_{i}}^{i}, c_{j_{i}}^{i}, e_{j_{i}}^{i}, f_{j_{i}}^{i}\right\} \cup \bigcup_{1 \leqslant i \leqslant n} \bigcup_{j \in \bar{J}_{i}}\left\{b_{j}^{i}, c_{j}^{i}, e_{j}^{i}, f_{j}^{i}, p_{j}^{i}, q_{j}^{i}\right\} \cup \underset{1 \leqslant j \leqslant m,\left|c_{j}\right|=2}{\bigcup}\left\{c_{j}^{n+1}\right\} .
$$

We finish the definition of the edge set by defining the edges incident to the vertices of the stable set $S$. We use the following convention: whenever we refer to a vertex $a_{t}^{i^{*}}, i$ belongs to $I_{t}=\left\{i_{1}, i_{2}, i_{3}\right\}$ with $i_{1}<i_{2}<i_{3}$, and $i^{*}$ is defined as follows:

$$
i^{*}= \begin{cases}i_{2}, & \text { if } i=i_{1}, \\ i_{3}, & \text { if } i=i_{2}, \\ i_{1}, & \text { if } i=i_{3} .\end{cases}
$$

For $1 \leqslant i \leqslant n, N\left(b_{j_{i}}^{i}\right)=\left\{a_{j_{i}}^{i^{*}}, d_{j_{i}}^{i}\right\}, N\left(c_{j_{i}}^{i}\right)=\left\{a_{j_{i}}^{i^{*}}, a_{j_{i}}^{i}, d_{j_{i}}^{i}\right\}, N\left(e_{j_{i}}^{i}\right)=\left\{d_{j_{i}}^{i}, h_{j_{i}}^{i}\right\}, N\left(f_{j_{i}}^{i}\right)=\left\{a_{j_{i}}^{i^{*}}, g_{j_{i}}^{i}\right\}$. For $1 \leqslant i \leqslant n, j \in \bar{J}_{i}, N\left(b_{j}^{i}\right)=$ $\left\{a_{j}^{i^{*}}, d_{j}^{i}\right\}, N\left(c_{j}^{i}\right)=\left\{a_{j}^{i^{*}}, a_{j}^{i}, d_{j}^{i}\right\}, N\left(e_{j}^{i}\right)=\left\{d_{j}^{i}, h_{j_{i}}^{i}\right\}, N\left(f_{j}^{i}\right)=\left\{a_{j}^{i^{*}}, g_{j_{i}}^{i}\right\}, N\left(p_{j}^{i}\right)=\left\{a_{j_{i}}^{i}, g_{j_{i}}^{i}, a_{j}^{i}\right\}, N\left(q_{j}^{i}\right)=\left\{a_{j_{i}}^{i}, h_{j_{i}}^{i}, a_{j}^{i}\right\}$. For $1 \leqslant j \leqslant m$, $\left|c_{j}\right|=2, N\left(c_{j}^{n+1}\right)=\left\{a_{j}^{n+1}, a_{j}^{(n+1)^{*}}\right\}$.

Note that the constructed instance $G_{I}$ is a 3-split graph. Notice that for each variable $u_{i}$, graph $G_{I}$ contains as induced subgraph, Truth Setting component $T_{i}$, the graph depicted in Fig. 1 for the case variable $u_{i}$ has 3 occurrences. Throughout the paper, we shall use the convention in the figures: vertices of $K$ are black, vertices of $S$ are white; only edges between vertices of the same cone are drawn which means all other edges between black vertices are omitted. 


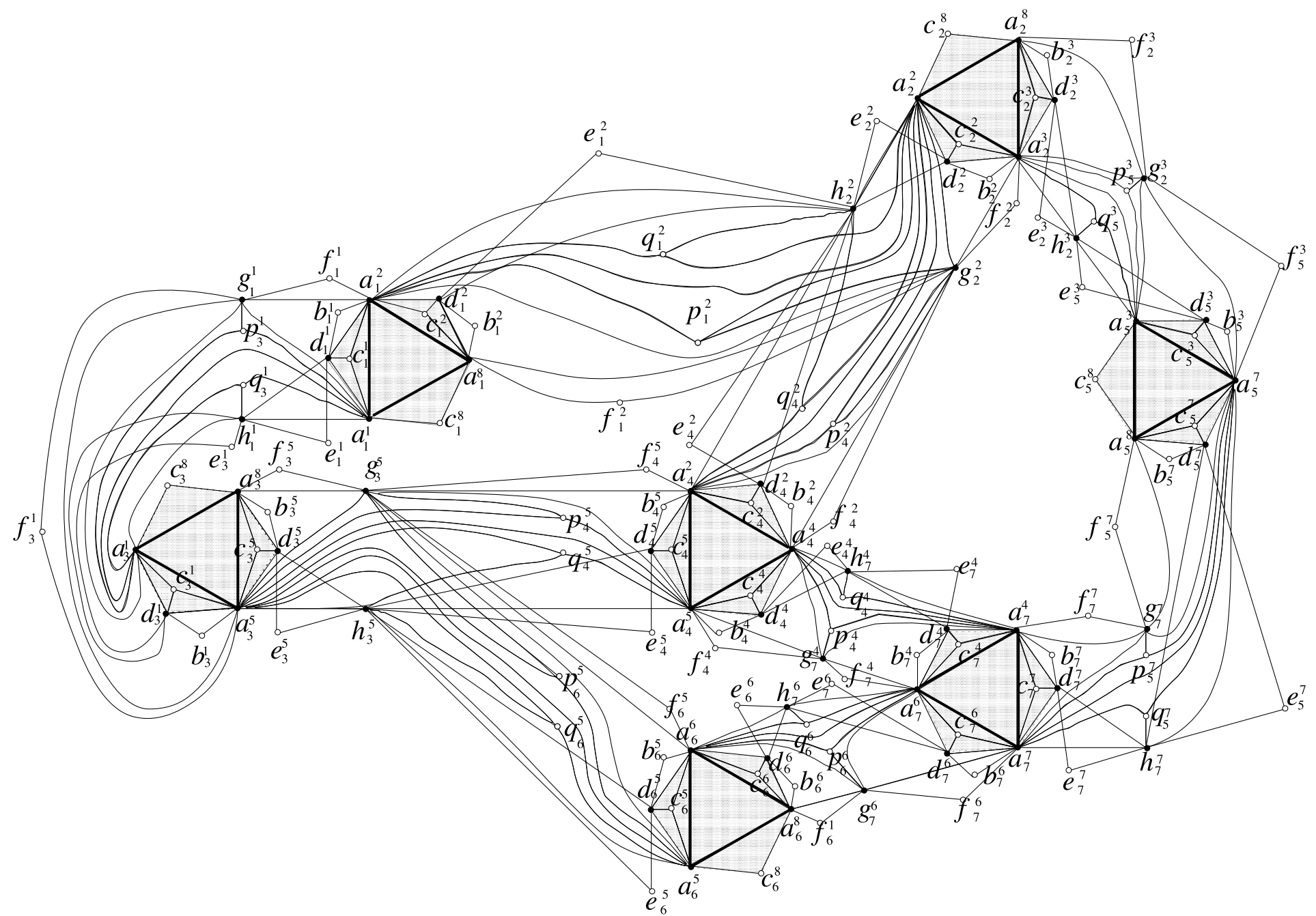



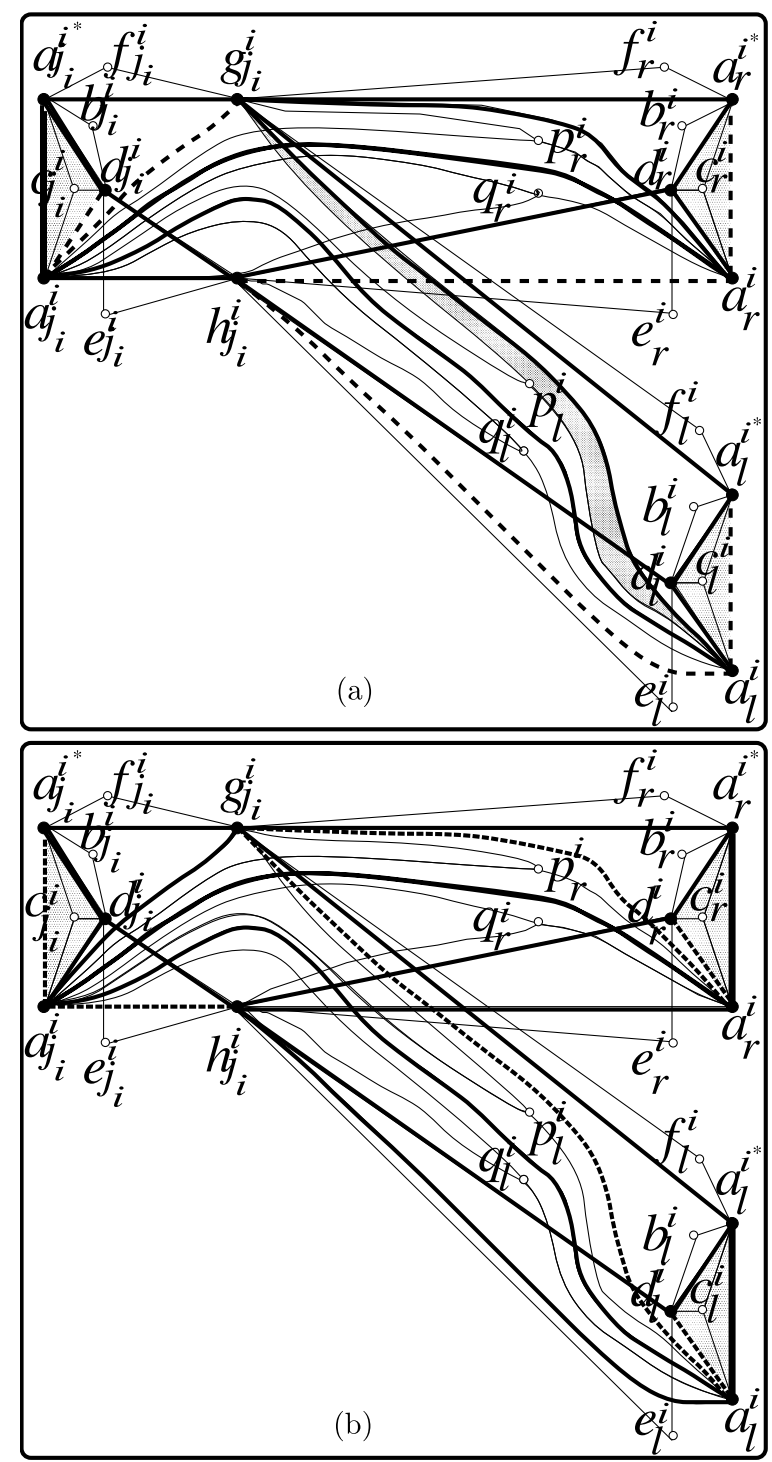

Fig. 3. (a) RS-basis for $T_{i}$ containing edge $a_{r}^{i} d_{r}^{i}$ is depicted in bold edges. Dashed edges are the edges of the bases of the 3-cones that are not members of the RS-basis. (b) Respectively for edge $a_{r}^{i} a_{r}^{i^{*}}$.

For the convenience of the reader, we present in Fig. 2 an example of the whole graph $G_{I}$ obtained from an instance $I$ of $3 \mathrm{SAT}_{\overline{3}}$.

We refer to Fig. 3 for the proof of Lemma 6.

Lemma 6 (True edge-false edge). Suppose $\mathcal{F}$ be an RS-basis of the constructed graph $G_{I}$. For each $j, 1 \leqslant j \leqslant m$, and for each $i \in I_{j}, i \neq$ $n+1$, exactly one of the edges $a_{j}^{i} a_{j}^{i^{*}}, a_{j}^{i} d_{j}^{i}$ belongs to $\mathcal{F}$. For each $i, 1 \leqslant i \leqslant n$, and for each $j \in \bar{J}_{i}$, if $a_{j}^{i} d_{j}^{i} \in \mathcal{F}$ then $a_{j_{i}}^{i} a_{j_{i}}^{i^{*}} \in \mathcal{F}$, and if $a_{j}^{i} a_{j}^{i} \in \mathcal{F}$ then $a_{j_{i}}^{i} d_{j_{i}}^{i} \in \mathcal{F}$.

Proof. Consider any $j, 1 \leqslant j \leqslant m$, and $i \in I_{j}, i \neq n+1$. Assume with no loss of generality, $j=j_{i}$. By considering the 2-cone $N\left[b_{j_{i}}^{i}\right.$, notice that edge $a_{j_{i}}^{i^{*}} d_{j_{i}}^{i}$ must belong to the RS-basis $\mathcal{F}$ which implies that both edges $a_{j_{i}}^{i} i_{j_{i}}^{i^{*}}$ and $a_{j_{i}}^{i} d_{j_{i}}^{i}$ cannot belong to $\mathcal{F}$, which implies that exactly one of the edges $a_{j_{i}}^{i} a_{j_{i}}^{i^{*}}, a_{j_{i}}^{i} d_{j_{i}}^{i}$ belongs to $\mathcal{F}$.

Consider any $i, 1 \leqslant i \leqslant n$, and $j \in \bar{J}_{i}=\{r, l\}$. Say $j=r$ and refer to Fig. 3(a). Notice that, edge $h_{j_{i}}^{i} d_{r}^{i}$ must belong to the RS-basis $\mathcal{F}$. Assume that $a_{r}^{i} d_{r}^{i} \in \mathcal{F}$. Then $a_{r}^{i} h_{j_{i}}^{i} \notin \mathcal{F}$, and so by considering the 3 -cone $N\left[q_{r}^{i}\right]$, edges $a_{r}^{i} a_{j_{i}}^{i}, h_{j_{i}}^{i} a_{j_{i}}^{i} \in \mathcal{F}$. Notice that edge $h_{j_{i}}^{i} d_{j_{i}}^{i}$ must belong to the RS-basis $\mathcal{F}$. Hence $a_{j_{i}}^{i} d_{j_{i}}^{i} \notin \mathcal{F}$, and so by the first statement, $a_{j_{i}}^{i} a_{j_{i}}^{i *} \in \mathcal{F}$. Assume that $a_{r}^{i} a_{r}^{i *} \in \mathcal{F}$ and refer to Fig. 3(b) to obtain an analogous reasoning. 
Lemma 6 is the key for the NP-completeness result. Given any variable $u_{i}$ and any clause $c_{j}$ where $u_{i}$ occurs, any RS-basis of $G_{I}$ is forced to choose exactly one of the edges $a_{j}^{i} a_{j}{ }^{*}, a_{j}^{i} d_{j}^{i}$. If $r \in \bar{J}_{i}$, then any RS-basis of $G_{I}$ is forced to choose different types of edges incident to vertices $a_{r}^{i}$ and $a_{j_{i}}^{i}$, respectively. If $r, \ell \in \bar{J}_{i}$, then any RS-basis of $G_{I}$ is forced to choose the same type of edges incident to vertices $a_{r}^{i}$ and $a_{\ell}^{i}$, respectively. The correspondence between the two possible truth assignments of variable $u_{i}$ and the two possible edges incident to vertex $a_{j_{i}}^{i}$ is clear.

\section{Theorem 7. SPLIT CLIQUE GRAPH is NP-complete.}

Proof. As explained above, SPLIT CLIQUE GRAPH belongs to NP [1,2].

Let $G_{I}$ be the constructed 3-split graph obtained from an instance $I=(U, C)$ of $3 \operatorname{sAT}_{\overline{3}}$. Suppose $G_{I}$ is a clique graph, and we exhibit a truth assignment for $U$ such that $C$ is satisfied. By Theorem 5 , let $\mathcal{F}$ be an RS-basis for $G_{I}$. Let $u_{i} \in U$ be a variable. Set $u_{i}$ equal to true if and only if edge $a_{j_{i}}^{i} d_{j_{i}}^{i} \in \mathcal{F}$. To see that this truth assignment for $U$ satisfies $C$ consider a clause $c_{j}$ and its corresponding triangle $\left\{a_{j}^{i}, a_{j}^{i^{*}}, a_{j}^{i * *}\right\}$. Since $\mathcal{F}$ induces a triangle-free subgraph of $G_{I}[K]$, there exists $i \in I_{j}$ such that the edge $a_{j}^{i} a_{j}^{i^{*}}$ is not a member of $\mathcal{F}$. Notice that $i \neq n+1$. By Lemma 6 , edge $a_{j}^{i} a_{j}^{i^{*}} \notin \mathcal{F}$ implies that edge $a_{j}^{i} d_{j}^{i} \in \mathcal{F}$. If $j=j_{i}$ then variable $u_{i}$ is true and clause $c_{j}$ is satisfied. If $j \neq j_{i}$, then $j \in \bar{J}_{i}$, by Lemma 6 edge $a_{j}^{i} d_{j}^{i} \in \mathcal{F}$ implies edge $a_{j_{i}}^{i} a_{j_{i}}^{i^{*}} \in \mathcal{F}$, and edge $a_{j_{i}}^{i} d_{j_{i}}^{i} \notin \mathcal{F}$. It follows that $u_{i}$ is false, and then $c_{j}$ is satisfied.

Conversely, given a truth assignment of $U$ that satisfies $C$, by Theorem 5 , it suffices to exhibit an RS-basis $\mathcal{F}$ in order to prove that $G_{I}$ is a clique graph. Let $\mathcal{F}$ contain:

For each $i, 1 \leqslant i \leqslant n$, the edges $a_{j_{i}}^{i^{*}} g_{j_{i}}^{i}, d_{j_{i}}^{i} h_{j_{i}}^{i}, a_{j_{i}}^{i^{*}} d_{j_{i}}^{i}$; and for each $j \in \bar{J}_{i}$, the edges $a_{j}^{i^{*}} g_{j_{i}}^{i}, d_{j}^{i} h_{j_{i}}^{i}, a_{j}^{i^{*}} d_{j}^{i}, a_{j_{i}}^{i} a_{j}^{i}$.

For each $j, 1 \leqslant j \leqslant m$ with $\left|c_{j}\right|=2$, the edges $a_{j}^{n+1^{*}} a_{j}^{n+1}$.

For each $i, 1 \leqslant i \leqslant n$, such that variable $u_{i}$ is true, the edges $d_{j_{i}}^{i} a_{j_{i}}^{i}, a_{j_{i}}^{i} g_{j_{i}}^{i}$; and for each $j \in \bar{J}_{i}$, the edges $h_{j_{i}}^{i} a_{j}^{i}$, $a_{j}^{i} a_{j}^{i^{*}}$.

For each $i, 1 \leqslant i \leqslant n$, such that variable $u_{i}$ is false, the edges $a_{j_{i}}^{i} a_{j_{i}}, a_{j_{i}}^{i} h_{j_{i}}^{i}$; and for each $j \in \bar{J}_{i}$, the edges $g_{j_{i}}^{i} a_{j}^{i}$, $a_{j}^{i} d_{j}^{i}$.

The proof is completed by showing that $\mathcal{F}$ indeed induces a triangle-free subgraph of $G_{I}[K]$ containing all the basis of 2-cones and two edges of the basis of each 3-cone. The latter is easily checked by looking at the list of cones of $G_{I}$ given in the construction of $G_{I}$. To see that there are no triangles, notice first that the edges included in the family above, for any fixed $i$, are exactly the bold edges in Fig. 3(a) if variable $u_{i}$ is false, and exactly the bold edges in Fig. 3(b) if variable $u_{i}$ is true. Therefore, they do not form any triangle. Thus, the only triangles that may have been formed by the edges of $\mathcal{F}$ are the triangles with vertices $a_{j}^{i_{1}}, a_{j}^{i_{2}}$ and $a_{j}^{i_{3}}$ for some $j$ and $I_{j}=\left\{i_{1}, i_{2}, i_{3}\right\}$ (see Fig. 2). But this implies that clause $c_{j}$ is not satisfied, a contradiction.

For the convenience of the reader, we present in Fig. 4 an example of an RS-family defined by a satisfying truth assignment, according to the proof of Theorem 7.

We remark that the split graph restriction produces the powerful concept of RS-basis of Theorem 5 which yields a much simpler proof when compared to the former clique graph NP-completeness proof. Note further that our NP-completeness result yields the following corollary:

\section{Corollary 8. SPANNING HELLY FAMILY is NP-complete.}

Proof. Given a set family $\mathcal{F}$, to obtain the split graph instance, we consider a complete set $K$ with vertices corresponding to the elements of the members of $\mathcal{F}$, a stable set $S$ with vertices corresponding to the members of $\mathcal{F}$, and set a vertex $x$ of $K$ adjacent to a vertex $\mathcal{F}$ of $S$ whenever $x \in \mathcal{F}$.

\subsection{3-Split graphs}

Denote by 3-split 3 the class of 3-split graphs such that the vertices of the independent set have degree exactly 3.

The NP-completeness proof for SPLIT CLIQUE GRAPH given in Theorem 7 constructs a graph instance that is a 3-split graph. We present next a recursive procedure that given a 3-split graph $G$ constructs in polynomial time a 3-split 3 graph $G_{H}$ in such a way that $G$ is a clique graph if and only if $G_{H}$ is a clique graph.

In order to avoid ambiguity, we denote the split partition for $V(G)$ as $(K(G), S(G))$, where $K(G)$ is the complete set and $S(G)$ is the independent set.

Let $G$ be a 3-split graph with 3-split partition $(K(G), S(G))$. Assume $s$ is a degree 2 vertex of $S(G)$ with $N(s)=\{u, w\}$.

Call $F_{S}$ the 3-split 3 graph such that $K\left(F_{S}\right)=\{u, w, 1,2,3,4,5,6,7,8\}$; and $S\left(F_{S}\right)$ has a vertex $z_{T}$ with neighborhood $N\left(z_{T}\right)=T$, for each triangle $T$ contained in any one of the subsets $\{u, w, 1,2\},\{1,2,3,4\},\{3,4,5,6\},\{5,6,7,8\}$, and $\{7,8,2, w\}$ of $K\left(F_{s}\right)$. Since each one of these subsets contains exactly 4 triangles, we have $\left|V\left(F_{s}\right)\right|=4 \times 5=20$.

In Fig. 5, we draw all the vertices of $K\left(F_{S}\right)$ and only the edges between them that define the triangles $T$ which are neighborhoods of the vertices $z_{T} \in S\left(F_{S}\right)$. In other words, each triangle $T$ defined by the edges drawn in Fig. 5 is the basis of the 3-cone of $F_{S}$ with extreme vertex $z_{T}$. Observe that $F_{S}$ has no other cones and that $V(G) \cap V\left(F_{S}\right)=\{u, w\}$. 


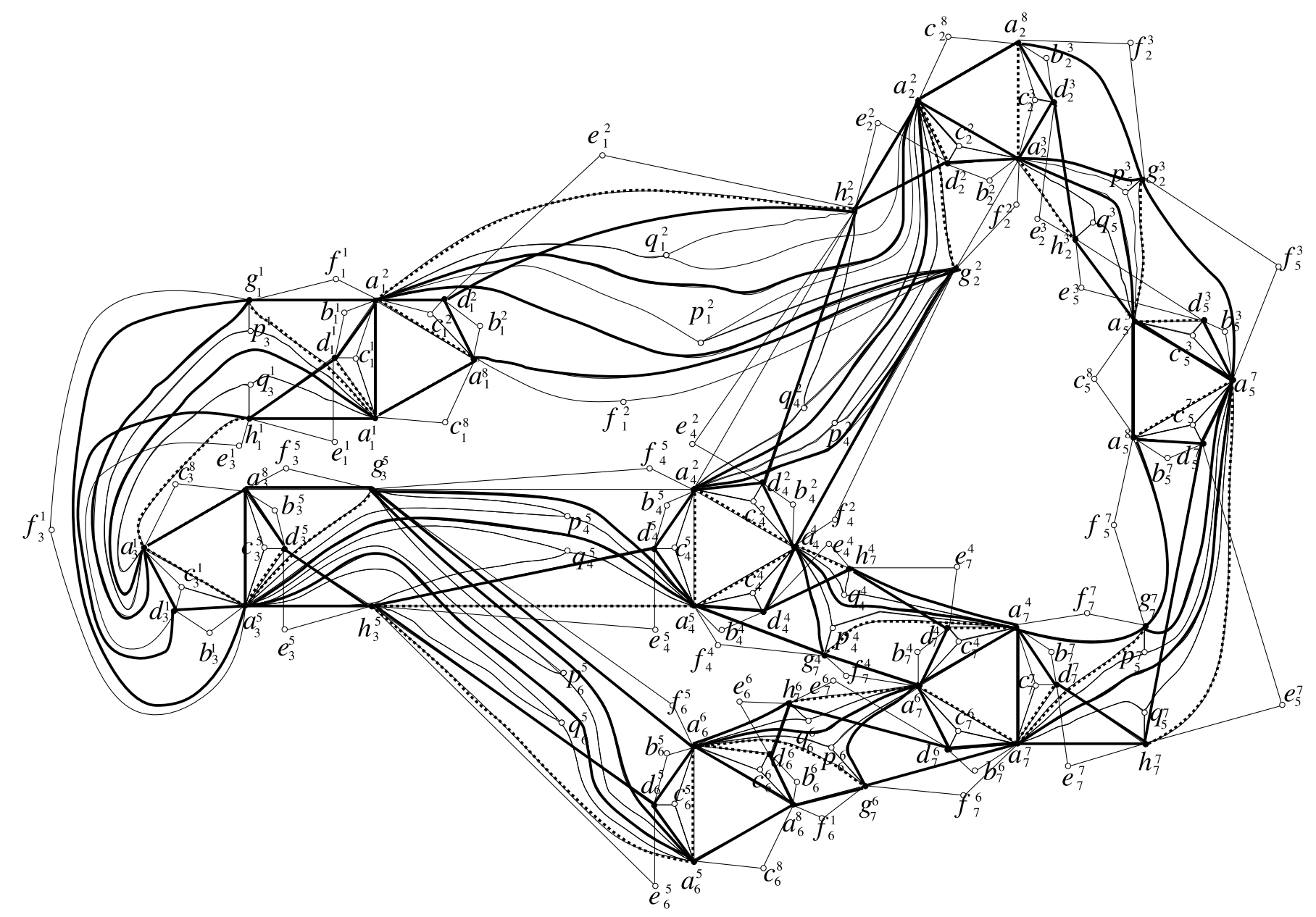

Fig. 4. Satisfying truth assignment for $3 \mathrm{sAT}_{\overline{3}}$ instance $I=(U, C)$, where $u_{1}=\bar{u}_{2}=u_{3}=\bar{u}_{4}=\bar{u}_{5}=u_{6}=\bar{u}_{7}=T$. 


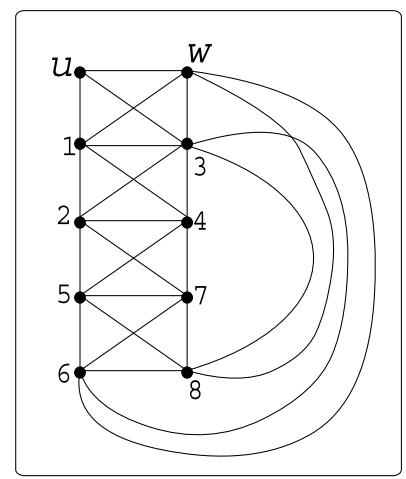

Fig. 5. Gadget $F_{s}$ : we draw only the vertices of $K\left(F_{s}\right)$, and only the edges that induce the triangle bases of a 3-cone.

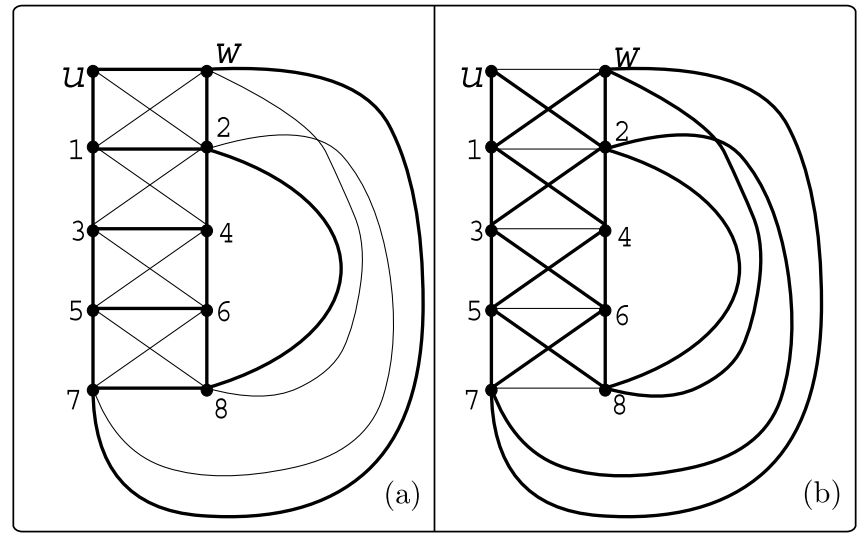

Fig. 6. (a) depicts RS-basis for graph $F_{s}$, and (b) is used for fact 3.

Finally, we obtain a graph $G_{s}$ by the union of $G-s$ and $F_{s}$ and by adding all necessary edges to make $K(G-s) \cup K\left(F_{s}\right)$ a complete set. Notice that $K(G-s)=K(G)$. Formally,

$$
\begin{aligned}
& V\left(G_{s}\right)=V(G-s) \cup V\left(F_{S}\right), \\
& E\left(G_{s}\right)=E(G-s) \cup E\left(F_{S}\right) \cup\{x y \mid x \in\{1,2,3,4,5,6,7,8\} \text { and } y \in K(G)-\{u, w\}\} .
\end{aligned}
$$

Observe that $G_{s}$ is a 3 -split graph with 3-split partition

$$
\begin{aligned}
& K\left(G_{S}\right)=K(G) \cup\{1,2,3,4,5,6,7,8\}, \\
& S\left(G_{s}\right)=(S(G)-\{s\}) \cup S\left(F_{S}\right) .
\end{aligned}
$$

Since each vertex in $S\left(F_{s}\right)$ has degree 3, the number of vertices of degree 2 of $S\left(G_{s}\right)$ is one less than the number of vertices of degree 2 of $S(G)$. It is clear that repeating the described procedure for each vertex of degree 2 of $S(G)$ we get a 3 -split 3 graph that will be called $G_{H}$ in the proof of Theorem 10.

To prove that $G_{s}$ is a clique graph if an only if $G$ is a clique graph, notice the following facts:

(1) The cones of $G_{s}$ are exactly the cones of $G-s$ plus the cones of $F_{s}$, thus the bases of the cones of $G_{s}$ are in $K(G)$ or in $K\left(F_{s}\right)$.

(2) The bold edges of $K\left(F_{s}\right)$ depicted in Fig. 6(a) form an RS-basis of $F_{s}$.

(3) Any RS-basis $B$ of $G_{s}$ contains the edge $u w$. Indeed, please refer to Fig. 6(b), if $u w$ is not in $B$, in order to contain the bases of exactly two triangles of the cone with extreme $z_{T}$ for $T=\{u, w, 1\}$, we have that $u 1,1 w$ must be in $B$. Analogously, in order to contain the bases of exactly two triangles of the cone with extreme $z_{T}$ for $T=\{u, w, 2\}$, we have that $u 2$ and $2 w$ must be in $B$. Now, in order to contain the bases of exactly two triangles of the cone with extreme $z_{T}$ for $T=\{1,2, u\}$, we have that 12 is not in $B$. Repeating this argument for each one of the $K_{4}$ 's $\{1,2,3,4\},\{3,4,5,6\}$, $\{5,6,7,8\}$, and $\{7,8,2, w\}$, we obtain that $2 w$ is not in $B$, which is a contradiction. 

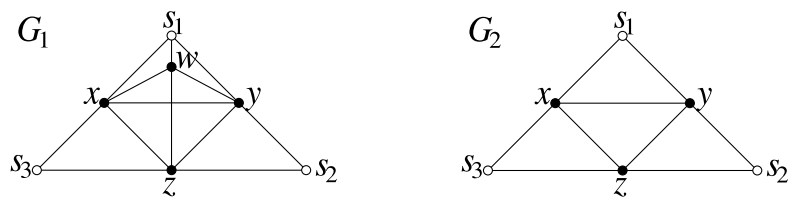

Fig. 7. (a) $w$ is a private neighbor of $s_{1}$. (b) No vertex in $S$ has a private neighbor.

Lemma 9. Graph $G_{s}$ is a clique graph if and only if $G$ is a clique graph.

Proof. By Theorem 5, since both graphs are 3-split, we have to show that there exists an RS-basis of $G_{s}$ if and only if there exists an RS-basis of $G$.

Let $B$ be an RS-basis of $G_{s}$. Consider the edge family $B^{\prime}$ formed by all the edges of $B$, contained in $K(G)$. Since the cones of $G$ are the cones of $G-s$ plus the 2-cone with extreme vertex $s$ and basis $u w$, by facts (1) and (3), $B^{\prime}$ is an RS-family of $G$.

Let $B$ be an RS-basis of $G$. Since $u w$ is the basis of the 2-cone with extreme $s$, we have that $u w$ belongs to $B$. Consider the RS-basis $B^{\prime}$ of $F_{S}$ depicted by the bold edges in Fig. 6(a). By fact (1), and since $u w$ belongs to $B$ and $B^{\prime}$, it follows that $B \cup B^{\prime}$ is an RS-family of $G_{s}$.

\section{Theorem 10. 3-SPLIT3 CLIQUE GRAPH is NP-complete.}

Proof. The problem is in NP because CLIQUE GRAPH is in NP.

We reduce the NP-complete problem 3-SPLIT CLIQUE GRAPH to 3-SPLIT3 CLIQUE GRAPH. Let G be an instance of 3-SPLIT CLIQUE GRAPH. We construct recursively an instance $G_{H}$ of 3-SPLIT3 CLIQUE GRAPH as described above. Since Lemma 9 can be applied at each step of the construction, it follows that $G$ is a clique graph if and only if $G_{H}$ is a clique graph.

\section{Polynomially solvable split clique graph classes}

In the following three theorems we present split graph subclasses for which clique graphs can be recognized in polynomial time. Theorem 11 defines a split graph subclass that is a split clique graph subclass. Theorems 12 and 13 define split graph subclasses that are non-trivial for the SPLIT CLIQUE GRAPH problem in the sense that both contain split clique graphs and non-split clique graphs.

In this section whenever we refer to the vertex subsets $K$ or $S$ of a split graph $G$, we mean the split partition $(K, S$ ) such that $K=\bigcup_{s \in S} N(s)$. Observe that for any split graph such partition always exists.

We say that a vertex $w \in K$ is a private neighbor of $s \in S$, if $s$ is the only vertex in $S$ adjacent to $w$, i.e. $N(w) \cap S=\{s\}$. We refer to Fig. 7.

Theorem 11. Let $G$ be a split graph. If every vertex $s \in S$ has a private neighbor then $G$ is a clique graph.

Proof. Suppose every vertex $s \in S$ has a private neighbor $h_{s}$. Let $x$ and $y$ be vertices of $K$. We say that $x$ is a twin of $y$ when $N[x]=N[y]$. Observe this is an equivalence relation, and so the equivalence classes define a partition of $K$. Let $R_{S}$ be the class of $h_{s}$ for $s \in S$; and $R_{1}, R_{2}, \ldots, R_{k}$ the remaining classes, this means the classes that do not contain any vertex $h_{s}$ for $s \in S$. We notice that $\left(\left(R_{S}\right)_{s \in S}, R_{1}, R_{2}, R_{3}, \ldots R_{k}\right)$ is a partition of $K$. Since $h_{s}$ is a private neighbor of $s$, if $s^{\prime} \in S$ and $s^{\prime} \neq s$ then $R_{s} \neq R_{S^{\prime}}$.

For every $s \in S$, we call $I_{S}$ the set $\left\{i: 1 \leqslant i \leqslant k\right.$ such that $\left.R_{i} \subseteq N(s)\right\}$. Let $\mathcal{F}$ be the family of complete sets of $G$ whose members are: $K ; F_{s, i}=R_{S} \cup R_{i} \cup\{s\}$, for each $s \in S, I_{s} \neq \emptyset$ and $i \in I_{s} ; F_{S}=R_{S} \cup\{s\}$, for each $s \in S, I_{s}=\emptyset$. We claim that $\mathcal{F}$ is an RS-family of $G$, and so $G$ is a clique graph.

Let $e \in E_{G}$. If both end vertices of $e$ are in $K$ then $e$ is covered by $K$ which is a member of $\mathcal{F}$. If not, since $S$ is a stable set, then $e=s x$ with $s \in S$ and $x \in K$. If there exists $i, 1 \leqslant i \leqslant k$, such that $x \in R_{i}$, since every vertex in $R_{i}$ is a twin of $x$ and $x$ is adjacent to $s$, then $R_{i} \subseteq N(s)$. It follows that $F_{s, i}$ covers $e=s x$.

If such $i$ does not exist, there must exist $s^{\prime} \in S$ such that $x \in R_{s^{\prime}}$. Then $N[x]=N\left[s^{\prime}\right]$ and, since $s x \in E$, it follows that $s=s^{\prime}$, and so $x \in R_{s}$. Thus $e$ is covered by $F_{s}$ or by $F_{s, i}$ for any $i$ such that $R_{i} \subseteq N(s)$.

To prove that $\mathcal{F}$ has the Helly property, notice the following facts:

(1) $F_{S}$ is not a member of $\mathcal{F}$ if and only if there exists $i$ such that $F_{s, i}$ is a member of $\mathcal{F}$.

(2) $F_{s, i} \cap F_{s^{\prime}, i^{\prime}} \neq \emptyset$ implies $i=i^{\prime}$ or $s=s^{\prime}$.

(3) $F_{S}$ has empty intersection with all members of $\mathcal{F}$ except $K$.

Now, assume $\mathcal{F}^{\prime}$ is a pairwise intersecting subfamily with at least three members. Consider the members that are not $K$. By fact (3), all of them must be of type $F_{s, i}$. Moreover, by fact (2), there must exist $i$ such that all these members have the same subindex $i$; or there must exist $s$ such that all of them have the same subindex $s$. In the first case, all members of $\mathcal{F}^{\prime}$ 


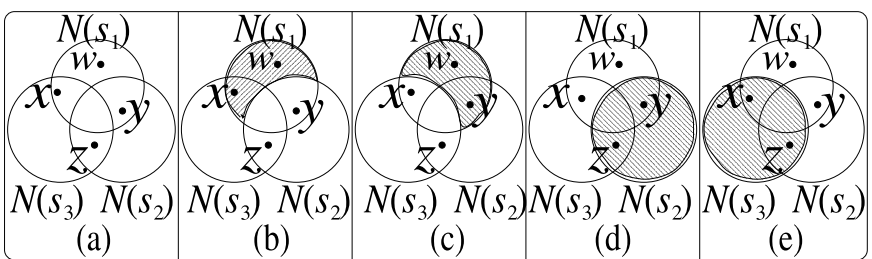

Fig. 8. Case in which $w$ is a private neighbor of $s_{1}$.

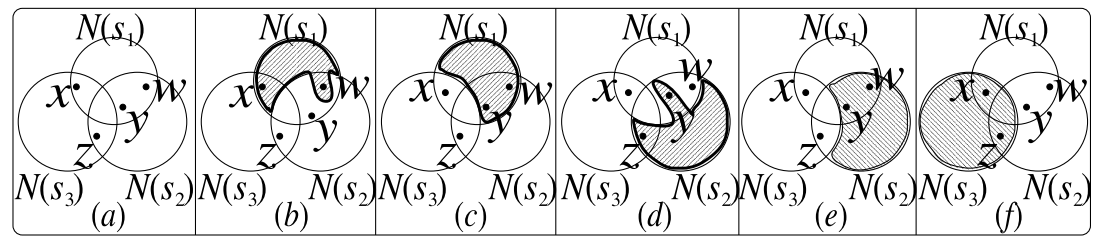

Fig. 9. Case in which $w$ is not a private neighbor.

have the vertices of $R_{i}$ in common. In the second case, all members of $\mathcal{F}^{\prime}$ have the vertices of $R_{S}$ in common. It follows that $\mathcal{F}^{\prime}$ has nonempty total intersection. This completes the proof.

Theorem 12. Let $G$ be a split graph with $|S| \leqslant 3$. Graph $G$ is not a clique graph if and only if there exist three 2-cones whose bases form a triangle.

Proof. Lemma 4 shows that if such three 2-cones exist then the graph $G$ is not a clique graph.

To prove the direct implication assume that $G$ is not a clique graph, thus the family of cliques of $G$ does not satisfy the Helly property. This implies that $|S|=3$, say $S=\left\{s_{1}, s_{2}, s_{3}\right\}$, and that $N\left[s_{1}\right], N\left[s_{2}\right]$ and $N\left[s_{3}\right]$ is a family of pairwise intersecting cliques of $G$ without a common vertex. Let $x, y$ and $z$ be vertices of $K$ such that $x \in N\left[s_{1}\right] \cap N\left[s_{3}\right], y \in N\left[s_{1}\right] \cap$ $N\left[s_{2}\right]$ and $z \in N\left[s_{2}\right] \cap N\left[s_{3}\right]$. If each $N\left[s_{i}\right]$ is a 2-cone then their bases form the triangle $\{x, y, z\}$ and the proof follows; otherwise, at least one of the $N\left[s_{i}\right]$ contains a third vertex $w$ of $K$; we will prove that this contradicts the fact that $G$ is not a clique graph.

Indeed, if $w$ is a private neighbor, without loss of generality assume of $s_{1}$, then it is easy to check that the complete set family

$$
N\left[s_{1}\right] \backslash N\left[s_{2}\right], \quad N\left[s_{1}\right] \backslash N\left[s_{3}\right], \quad N\left[s_{2}\right], N\left[s_{3}\right] \text { and } K
$$

(see Fig. 8) satisfies the conditions of Theorem 1 , thus $G$ is a clique graph.

If $w$ is not a private neighbor, without loss of generality assume $w \in N\left(s_{1}\right) \cap N\left(s_{2}\right)$ and $N\left(s_{i}\right) \backslash\left(N\left(s_{j}\right) \cup N\left(s_{k}\right)\right)=\emptyset$ for the three different possible sub-indices. In this case, the complete set family

$$
\begin{aligned}
& N\left[s_{1}\right] \backslash\left(N\left[s_{2}\right]-\{w\}\right), \quad N\left[s_{1}\right] \backslash N\left[s_{3}\right], \quad N\left[s_{2}\right] \backslash\left(N\left[s_{1}\right]-\{y\}\right) \\
& N\left[s_{2}\right] \backslash N\left[s_{3}\right], \quad N\left[s_{3}\right] \quad \text { and } K
\end{aligned}
$$

(see Fig. 9) satisfies the conditions given by Theorem 1 , so $G$ is a clique graph. Observe that if $N\left[s_{1}\right] \backslash\left(N\left[s_{2}\right]-\{w\}\right)$ (Fig. 9(b)) belongs to a pairwise intersecting family $\mathcal{F}^{\prime}$, then $N\left[s_{2}\right] \backslash\left(N\left[s_{1}\right]-\{y\}\right) \notin \mathcal{F}^{\prime}$ (Fig. 9(d)). The same occurs between $N\left[s_{1}\right] \backslash N\left[s_{3}\right]$ (Fig. 9(c)) and $N\left[s_{3}\right]$ (Fig. 9(f)). Hence, three intersecting complete sets of $\mathcal{F}^{\prime}$ have $x$, or $z$, or $w$ as a common element. The proof is complete.

Theorem 13. Let $G$ be a split graph with $|K| \leqslant 4$. Graph $G$ is not a clique graph if and only if: (i) there exist three 2-cones whose bases form a triangle; or (ii) there exist one 3 -cone and three 2 -cones whose bases are respectively $\{x, y, w\},\{x, z\},\{y, z\}$ and $\{z, w\}$, being $x, y, z, w$ vertices of $K$.

Proof. Lemma 4 shows that each one of the conditions (i) and (ii) implies $G$ is not a clique graph.

To prove the direct implication assume that $G$ is not a clique graph. Therefore, the clique family of $G$ has not the Helly property, which implies that some of the four ocular graphs in Fig. 10 is an induced subgraph of $G$ [16]. Since $G$ is split, $G$ has no induced cycles of size at least 4, it follows that none of the graphs $H_{1}, H_{2}, H_{3}$ is an induced subgraph of $G$. Thus, $H_{4}$ is an induced subgraph of $G$. Label the vertices of $H_{4}$ as in Fig. 7 (b); it is clear that $s_{1}, s_{2}$ and $s_{3}$ belong to $S$ and that $x, y$ and $z$ belong to $K$. If $N\left(s_{1}\right)=\{x, y\}, N\left(s_{2}\right)=\{y, z\}$ and $N\left(s_{3}\right)=\{x, z\}$, then there are three 2-cones satisfying (i) and the proof follows. Otherwise, without loss of generality, we can assume that there exists a vertex $w \in K \backslash\{x, y, z\}$ such that $s_{1}$ is adjacent to $w$; even more, we can assume that every vertex of $S$ adjacent to $x$ and $y$ and non-adjacent to $z$ is adjacent to $w$, see Fig. 7(a). 


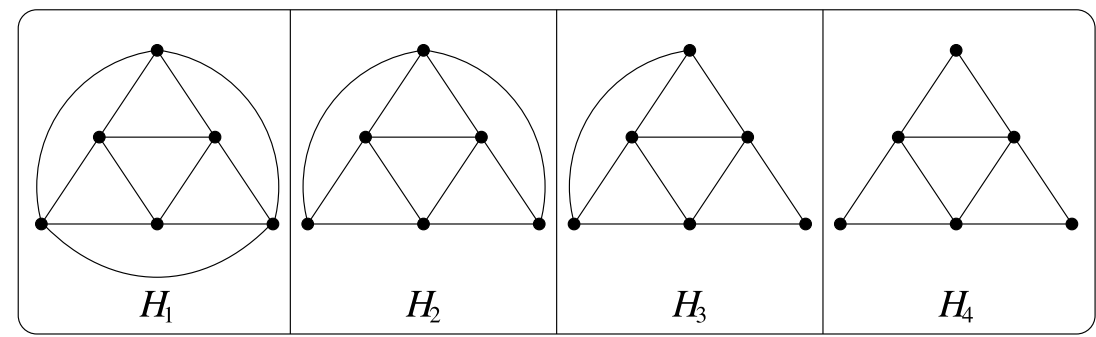

Fig. 10. Forbidden induced subgraphs for the class hereditary clique Helly.

Notice that $N\left(s_{1}\right)=\{x, y, w\}$ is a 3-cone; we will prove that there exist three 2-cones with bases $=\{z, x\},\{z, w\}$ and $\{z, y\}$ respectively, thus (ii) holds. Without loss of generality, assume for a contradiction that there is no 2-cone with basis $\{z, w\}$; thus every vertex $S$ of $S$ adjacent to $z$ and $w$ is also adjacent to $x$ or to $y$. We claim that in such a case $G$ is a clique graph, a contradiction.

Indeed, we will show that the following set family $\mathcal{F}$ is an RS-family of $G$. The members of $\mathcal{F}$ are:

$K$;

$N[s] \backslash\{x\}$ and $N[s] \backslash\{y\}$ whenever $N[s]$ is a 3-cone and $\{x, y\} \subseteq N[s]$;

$N[s] \backslash\{z\}$ and $N[s] \backslash\{w\}$ whenever $N[s]$ is a 3-cone and $\{z, w\} \subseteq N[s]$;

$N[s]$ for any other $s \in S$.

Notice that

$$
\begin{aligned}
& F \in \mathcal{F} \text { and } z \notin F \Rightarrow F \cap K=\{x, w\} \text { or } F \cap K=\{y, w\}, \\
& F \in \mathcal{F} \text { and } w \notin F \Rightarrow F \cap K=\{x, z\} \text { or } F \cap K=\{y, z\}, \\
& F \in \mathcal{F} \text { and } x \notin F \Rightarrow F \cap K=\{y, w\} \text { or } F \cap K=\{y, z\}, \\
& F, F^{\prime} \in \mathcal{F} \text { and } F \cap F^{\prime} \neq \varnothing \Rightarrow F \cap F^{\prime} \cap K \neq \emptyset .
\end{aligned}
$$

It is clear that the members of $\mathcal{F}$ are complete sets of $G$ and that they cover all the edges. Let us see that $\mathcal{F}$ has the Helly property. Assume for a contradiction that there exists a pairwise intersecting subfamily $\mathcal{F}^{\prime}$ without a common vertex. Thus there exist members $F_{1}$ and $F_{2}$ such that

$$
z \notin F_{1} \quad \text { and } \quad w \notin F_{2} \text {. }
$$

By the previous implication (1), $F_{1}$ and $F_{2}$ are two different members of $\mathcal{F}^{\prime}$ and we can assume without loss of generality that $F_{1} \cap K=\{x, w\}$. Since $w \notin F_{2}$ and $F_{1} \cap F_{2} \neq \emptyset$, it follows from implications (4) and (2), that $F_{2} \cap K=\{x, z\}$.

Finally, assume there exists a member $F_{3}$ of $\mathcal{F}^{\prime}$ such that $x \notin F_{3}$. Since $F_{3}$ intersects $F_{1}$ and $F_{2}$, we obtain that $\{z, w\} \subseteq$ $F_{3} \cap K$ which contradicts implication (3). It follows that every member of $\mathcal{F}^{\prime}$ contains the vertex $x$. It contradicts the assumption that the $\mathcal{F}^{\prime}$ has not a common vertex. The proof is complete.

\section{Open related problems}

We summarize in a table the results and open problems we have managed to state about the complexity of the problem of recognizing clique graphs when restricted to split graphs. Denote by 3-split ${ }_{3}$ the class of 3-split graphs, where the vertices of the independent set have degree exactly 3.

\begin{tabular}{|c|c|c|c|c|c|c|c|}
\hline & 3-split & 3-split & $\begin{array}{c}\forall s \in S, s \text { has a } \\
\text { private neighbor }\end{array}$ & \multicolumn{2}{|c|}{$\begin{array}{c}|S| \\
\text { bounded }\end{array}$} & \multicolumn{2}{|c|}{$\begin{array}{c}|K| \\
\text { bounded }\end{array}$} \\
\hline $\begin{array}{c}\text { Split graph } \\
G=(V, E) \\
\text { partition }(K, S)\end{array}$ & NPC & NPC & $\mathrm{P}$ & $\frac{|S| \leqslant 3}{\mathrm{P}}$ & general & $\frac{|K| \leqslant 4}{\mathrm{P}}$ & general \\
\hline
\end{tabular}

The present work defines three distinct sufficient conditions for a split graph to be a clique graph that lead to three polynomial split clique graph classes. The complexity of recognizing split clique graphs with $|K|$ or $|S|$ bounded remains open.

Several subclasses of clique graphs have been studied for which polynomial-time recognition is known. In particular, for several classes of graphs the corresponding class of clique graphs is known [23]. Note that it is well known that the clique graph of a chordal graph is a dually chordal graph $[5,21]$ but the complexity of deciding whether a chordal graph is a clique graph was a challenging open problem. We have proved that deciding whether a given chordal graph is a clique graph is an 
NP-complete problem since the class of split graphs is contained in the class of chordal graphs. CLIQUE GRAPH was proposed as a possible complexity-separating problem for the classes of chordal graphs and split graphs [8], and we have proved that it is not.

The NP-completeness of CLIQUE GRAPH [1,2] suggested the study of the problem restricted to classes of graphs not properly contained in the class of clique graphs. One such class is the class of split graphs, the object of the present paper, and the recognition of split clique graphs is proved NP-complete. Another challenging still open problem is the recognition of planar clique graphs [3].

Let $G$ be a split graph with split partition $(K, S)$. In case $G$ is a 3-split graph, Theorem 5 says $G$ admits an RS-family containing $K$. We leave as open the complexity of deciding if a split clique graph with split partition (K, $S$ ) admits an RS-family containing $K$.

Our NP-completeness result for split clique graph recognition is optimum in the sense that each vertex of the independent set of our split instance has degree at most 3, whereas when each vertex of the independent set has degree at most 2 the problem is polynomial, since it is reduced to check whether the clique family of the graph satisfies the Helly property. Actually, by Theorem 5 the problem is polynomial when the input is a 3-split graph such that the number of 3-cones is bounded, which implies that 3-split clique graph recognition when $|K|$ is bounded or when $|S|$ is bounded is in P. Our additional NP-completeness result proves that the problem is NP-complete even when each vertex of the independent set has degree exactly 3 . Note that the problem $3 \mathrm{SAT}_{\overline{3}}$ when restricted to having exactly three literals per clause is polynomial [14].

\section{References}

[1] L. Alcón, L. Faria, C.M.H. de Figueiredo, M. Gutierrez, Clique graph recognition is NP-complete, in: Proceedings of Graph-Theoretic Concepts in Computer Science, WG'06, in: Lecture Notes in Comput. Sci., vol. 4271, Springer, 2006, pp. 269-277.

[2] L. Alcón, L. Faria, C.M.H. de Figueiredo, M. Gutierrez, The complexity of clique graph recognition, Theoret. Comput. Sci. 410 (2009) 2072-2083.

[3] L. Alcón, M. Gutierrez, Cliques and extended triangles. A necessary condition for planar clique graphs, Discrete Appl. Math. 141 (2004) 3-17.

[4] A. Bondy, G. Durán, M.C. Lin, J.L. Szwarcfiter, Self-clique graphs and matrix permutations, J. Graph Theory 44 (2003) $178-192$.

[5] A. Brandstädt, F.F. Dragan, V.D. Chepoi, V. Voloshin, Dually chordal graphs, SIAM J. Discrete Math. 11 (1998) $437-455$.

[6] A. Brandstädt, V.B. Le, J.P. Spinrad, Graph Classes: A Survey, SIAM Monographs on Discrete Mathematics and Applications, 1999.

[7] M.C. Dourado, F. Protti, J.L. Szwarcfiter, Complexity aspects of the Helly property: graphs and hypergraphs, Electron. J. Combin. 17 (2009) 1-53.

[8] C.M.H. de Figueiredo, The P vs. NP-complete dichotomy of some challenging problems in graph theory, Discrete Appl. Math. 160 (2012) 2681-2693.

[9] R.C. Hamelink, A partial characterization of clique graphs, J. Combin. Theory Ser. B 5 (1968) 192-197.

[10] F. Larrión, C.P. de Mello, A. Morgana, V. Neumann-Lara, M.A. Pizaña, The clique operator on cographs and serial graphs, Discrete Math. 282 (2004) $183-191$.

[11] F. Larrión, V. Neumann-Lara, M.A. Pizaña, Graph relations, clique divergence and surface triangulations, J. Graph Theory 51 (2006) $110-122$.

[12] M.C. Lin, F. Soulignac, J.L. Szwarcfiter, The clique operator on circular-arc graphs, Discrete Appl. Math. 158 (2010) 1259-1267.

[13] T.A. McKee, F.R. McMorris, Topics in Intersection Graph Theory, SIAM Monographs on Discrete Mathematics and Applications, 1999.

[14] C.H. Papadimitriou, Computational Complexity, Addison-Wesley, 1994

[15] C. Picinin de Mello, C. Lucchesi, J.L. Szwarcfiter, On clique-complete graphs, Discrete Math. 183 (1998) $247-254$.

[16] E. Prisner, Hereditary clique-Helly graphs, J. Combin. Math. Combin. Comput. 14 (1993) 216-220.

[17] E. Prisner, Graph Dynamics, Pitman Research Notes in Mathematics, vol. 338, Longman, 1995.

[18] E. Prisner, J.L. Szwarcfiter, Recognizing clique graphs of directed and rooted path graphs, Discrete Appl. Math. 94 (1999) $321-328$.

[19] F. Protti, J.L. Szwarcfiter, Clique-inverse graphs of $K_{3}$-free and $K_{4}$-free graphs, J. Graph Theory 35 (2000) $257-272$.

[20] F.S. Roberts, J.H. Spencer, A characterization of clique graphs, J. Combin. Theory Ser. B 10 (1971) 102-108.

[21] J.L. Szwarcfiter, C.F. Bornstein, Clique graphs of chordal graphs and path graphs, SIAM J. Discrete Math. 7 (1994) $331-336$.

[22] J.L. Szwarcfiter, Recognizing clique-Helly graphs, Ars Combin. 45 (1997) 29-32.

[23] J.L. Szwarcfiter, A survey on clique graphs, in: C. Linhares-Sales, B. Reed (Eds.), Recent Advances in Algorithms and Combinatorics, in: CMS Books Math. (Ouvrages Math. SMC), vol. 11, Springer, New York, 2003, pp. 109-136. 